\title{
First Irish delivery following sequential, two-stage embryo and blastocyst transfer
}

\author{
L. H. Hayrinen - E. S. Sills • A. O. Fogarty • \\ D. J. Walsh • A. D. Lutsyk • A. P. H. Walsh
}

Received: 2 February 2009/Accepted: 25 August 2009/Published online: 8 October 2009

(C) The Author(s) 2009. This article is published with open access at Springerlink.com

\begin{abstract}
Background The timing of embryo transfer (ET) after in vitro fertilisation (IVF) remains controversial, and there are no reliable guidelines available to prospectively identify which patients would benefit from either day-3 or blastocyst transfer. While blastocyst transfer is generally favoured over day-3 transfers, very few IVF patients get both in the same treatment cycle.

Case description We report on a 35.5-year-old female with tubal factor infertility who underwent IVF, which included transfer of a fresh day-3 embryo and a thawed blastocyst frozen at day 6. Transfer occurred on two separate days (days 3 and 6) in a two-stage/dual catheter fashion and resulted in a healthy term singleton livebirth. Conclusions While combined day-3 and day-5 ET has been available elsewhere for several years, this is the first description of its successful application in Ireland and confirms the effectiveness of coordinated two-stage transfer in a single IVF treatment cycle.
\end{abstract}

Keywords Embryo transfer - Sequential - Two stage · Blastocyst

\footnotetext{
L. H. Hayrinen

Department of Obstetrics and Gynaecology, School of Medicine, Royal College of Surgeons in Ireland, Dublin, Ireland

E. S. Sills $(\bowtie) \cdot$ A. O. Fogarty · D. J. Walsh · A. P. H. Walsh The Sims Institute/Sims International Fertility Clinic, Rosemount Hall, Dundrum Road, Dundrum, Dublin 14, Ireland e-mail: drscottsills@sims.ie
}

\section{A. D. Lutsyk}

Department of Cell Biology, Histology and Embryology,

School of Medicine, Lviv National Medical University, Lviv, Ukraine

\section{Introduction}

Embryo transfer (ET) is typically a single-step process where all embryos destined for the uterus are placed by one catheter. The timing of ET has long been controversial, but as the human embryo normally arrives inside the uterine compartment from the oviduct at the blastocyst stage, many authorities have advocated this as a more natural and physiologically preferred approach, superior to day $3 \mathrm{ET}$. Moreover, when transferred at day 2 or 3, embryo development is directed by maternal transcripts and stored mRNA exclusively from the oocyte only, because the embryonic genome remains latent at this time. Although blastocyst transfer has distinct advantages over earlier transfer protocols in in vitro fertilisation (IVF), intentionally culturing all embryos to blastocyst stage brings the risk of cancelling the transfer should no embryos survive in culture. To avoid this unfortunate outcome, a strategy of consecutive (two stage) ET has been described $[1,2]$ where the patient first has a day-3 transfer followed by a second (blastocyst) transfer in the same cycle-usually 3 days later. Although deployed elsewhere for several years, this two-stage approach to ET for IVF patients has not been reported in Ireland until now.

\section{Case report}

A 35.5-year-old non-smoking Caucasian $\mathrm{G}_{2} \mathrm{P}_{1011}$ attended for reproductive endocrinology consultation with a history of bilateral tubal disease and unilateral oophorectomy. Two years previously, the patient underwent IVF with a two-ET (at day 3) which resulted in a healthy singleton term livebirth; that treatment cycle also produced two embryos which were cryopreserved at day 3 but did not survive 
thaw. She returned for a second fresh IVF cycle, but follicular recruitment was poor and only one blastocyst was available for transfer. Unfortunately, her transfer was cancelled secondary to an intrauterine fluid collection identified on transvaginal ultrasound. The single blastocyst was frozen at day 6 with spontaneous resolution of the endometrial fluid the following week.

The patient elected to begin a third fresh IVF cycle 2 months later, using a combined $600 \mathrm{IU} /$ day FSH (Puregon ${ }^{\circledR}$, Organon (Ireland) Ltd; Swords, Co. Dublin) + 75 IU/day LH (Luveris ${ }^{\circledR}$, MSD Serono Ltd; Middlesex, UK) ovulation induction protocol beginning on cycle day 2 without pituitary downregulation. Based on the endocrine status and follicular growth monitoring, we initiated $0.25 \mathrm{mg} /$ day GnRH-antagonist (Orgalutran $^{\circledR}$, Organon (Ireland) Ltd; Swords, Co. Dublin) to suppress native LH activity on day 9. Five oocytes were retrieved after a 12-day follicular recruitment phase, but only one advanced to the $2 p n$ stage following intracytoplasmic sperm injection. This single eight-cell embryo was transferred fresh under transabdominal ultrasound guidance on day 3; the remaining cryopreserved blastocyst was thawed next and transferred on day 6. A positive pregnancy test was noted 12 days after the second-stage transfer and serial hCG determinations were consistent with a normal pregnancy. Early obstetrical ultrasound was performed on day 55 and two distinct intrauterine implantation sites were noted. These structures were asymmetric and the smaller sac appeared empty. Follow-up sonograms confirmed the regression of the smaller sac (see Fig. 1), and the larger gestational sac included a foetal pole with a heart rate of 110 per min. Combined estradiol/progesterone support was continued until the 12th week. An uncomplicated

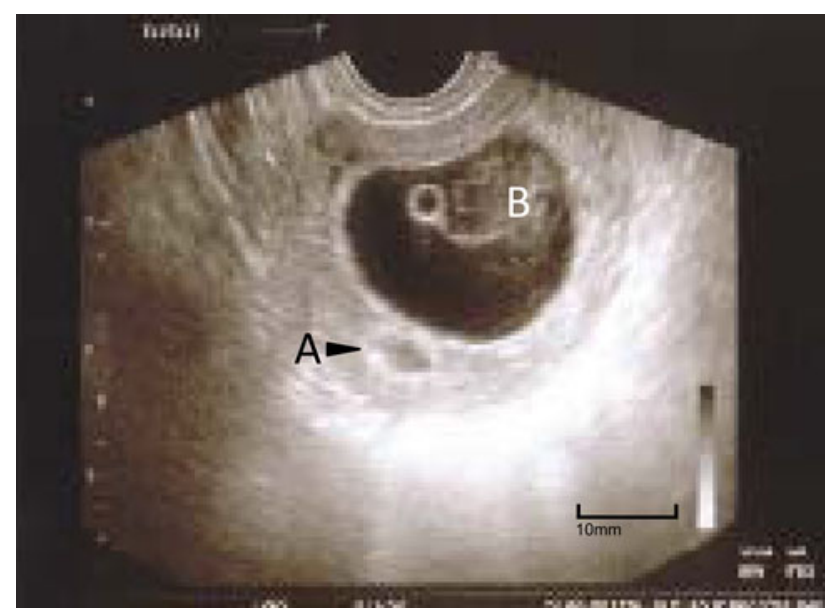

Fig. 1 Early pregnancy with regression of intrauterine implantation site (a) and viable gestational sac (b) resulting from two-stage, consecutive embryo transfer, as seen on transvaginal ultrasound at 8.5 weeks' gestation obstetrical course followed, and a healthy male infant (birth weight $=3,913 \mathrm{~g}$ ) was delivered at 40 2/7 weeks' gestation by Cesarian. Mother and baby were discharged home in good condition after 5 days; both continue to do well.

\section{Discussion}

Embryo transfer is the final and perhaps most critical step in determining IVF outcome. Indeed, it has been estimated that poor ET technique may account for many IVF failures-perhaps up to $30 \%$ of unsuccessful cycles [3]. It is generally recognised that the skill of the clinician performing ET is more important than catheter type or day of transfer, although most ETs in IVF are done as one procedure where the uterine cavity receives all cultured embryos from a single catheter at the same time. However, recent work has suggested that a two-stage ET specifically for patients with prior IVF failure can yield significantly higher pregnancy rates compared with those having traditional one-step ET $[4,5]$. Which ET procedure resulted in the ongoing singleton pregnancy cannot be known with certainty in our patient, but since allied research has suggested that performing an endometrial biopsy prior to actual transfer induces a favourable endometrial response [6], we speculate that our patient's viable pregnancy may be a continuation of the blastocyst transfer.

In this report, we confirm the effectiveness of coordinated two-stage transfer in a single cycle. Considerable multi-centre data have shown that offering extended in vitro culture and blastocyst transfer, whenever possible, can provide significantly improved pregnancy rates compared with traditional day-3 transfer [7, 8]. Day-3 transfer can still be appropriate for some patients, but there are no reliable guidelines available to prospectively identify which patients would benefit from each treatment approach [9]. Anticipating blastocyst transfer for every IVF patient is inappropriate and risks cycle cancellation due to culture failure, although we are unaware of any published data identifying unsuccessful blastocyst culture as a specific stressor causing patients to abandon IVF. Nevertheless, patient education and counselling are a crucial component of the pre-treatment programme for IVF. Although blastocyst transfer is generally favoured at our institution, day 3 transfers are still performed when clinically indicated. Very few IVF patients get both in the same treatment cycle, however. The current case illustrates a plan tailored to meet the disparate physiological needs of very different embryos, and presents the first published report of a delivery in Ireland after sequential ETs. Additional studies are underway to evaluate this treatment approach for other couples referred for prior IVF failure. 
Open Access This article is distributed under the terms of the Creative Commons Attribution Noncommercial License which permits any noncommercial use, distribution, and reproduction in any medium, provided the original author(s) and source are credited.

\section{References}

1. Goto S, Takebayashi K, Shiotani M, Fujiwara M, Hirose M, Noda Y (2003) Effectiveness of 2-step (consecutive) embryo transfer (comparison with cleavage-stage transfer). J Reprod Med 48:370-374

2. Phillips SJ, Dean NL, Buckett WM, Tan SL (2003) Consecutive transfer of day 3 embryos and of day 5-6 blastocysts increases overall pregnancy rates associated with blastocyst culture. J Assist Reprod Genet 20:461-464

3. Vereczkey A, Balyi B, Berkes E, Kovacs GT (2008) The impact of the embryo transfer catheter on the pregnancy rate. In: Allahbadia G (ed) Embryo transfer. Anshan Press, UK, pp 22-23
4. Almog B, Levin I, Wagman I et al (2008) Interval double transfer improves treatment success in patients with repeated IVF/ET failures. J Assist Reprod Genet 25(8):353-357

5. Esfandiari N, Coogan-Prewer J, Gotlieb L, Claessens EA, Casper RF (2008) Successful pregnancy following double-frozen embryo transfer in a patient with repeated implantation failure. Fertil Steril 90(4):1199.e13-15

6. Machtinger R, Dor J, Margolin M et al (2006) Sequential transfer of day 3 embryo and blastocysts after previous IVF failures despite adequate ovarian response. Reprod Biomed Online 13:376-379

7. Zhou L, Li R, Wang R, Huang HX, Zhong K (2008) Local injury to the endometrium in controlled ovarian hyperstimulation cycles improves implantation rates. Fertil Steril 89:1166-1176

8. de los Santos MJ, Mercader A, Galan A, Albert C, Romero JL, Pellicer A (2003) Implantation rates after two, three, or five days of embryo culture. Placenta Suppl B:S13-S19

9. Blake DA, Farquhar CM, Johnson N, Proctor M (2007) Cleavage stage versus blastocyst stage embryo transfer in assisted conception. Cochrane Database Syst Rev 4:CD002118 